\title{
High-Quality Immunofluorescence of Cultured Cells
}

\author{
Dibyendu Bhattacharyya, \\ Dibyendu Bhattacharyya, Ph.D., Department of Molecular Genetics and Cell Biology, The University \\ of Chicago, 920 East 58th Street, Chicago, IL 60615
}

Adam T. Hammond, and

Institute for Biophysical Dynamics, The University of Chicago, 929 East 57th St., Chicago, IL 60637

Benjamin S. Glick ${ }^{*}$

Department of Molecular Genetics and Cell Biology, The University of Chicago, 920 East 58th

Street, Chicago, IL 60615

\begin{abstract}
Immunofluorescence microscopy of cultured cells often gives poor preservation of delicate structures. We have obtained dramatically improved results with a simple modification of a standard protocol. Cells growing on a coverslip are rapidly dehydrated in a cold organic solvent, and then are rehydrated in a solution containing a homobifunctional crosslinker. The crosslinking reaction stabilizes cellular structures during subsequent incubation and wash steps, usually without compromising antigenicity. This method reproducibly yields high-quality images of endomembrane compartments and cytoskeletal elements.
\end{abstract}

\section{Keywords}

Immunofluorescence; Formaldehyde; Paraformaldehyde; Methanol; Acetone; Organic Solvents; Crosslinking; Transitional ER; ER Exit Sites; ER Export Sites

\section{Introduction}

Studies of the secretory pathway rely on methods to determine the intracellular locations of secretory cargo proteins and of trafficking machinery components. A common approach is to overexpress fluorescently tagged proteins, but both overexpression and tagging can cause aberrant localization. Immunofluorescence microscopy of cultured cells avoids this limitation by detecting endogenous proteins at their normal expression levels [1].

Unfortunately, the quality of immunofluorescence images is often poor. This problem can be ascribed to the gentle fixation procedures that are used to retain antigenicity. The most commonly used fixative is formaldehyde [1]. However, when we fixed cultured mammalian cells with formaldehyde to visualize transitional ER sites (tER sites; also known as ER exit sites), much of the information was lost. As judged by GFP tagging, discrete tER sites were present in the cell periphery and concentrated in the juxtanuclear Golgi region [2,3]; yet when tER sites were viewed by immunofluorescence microscopy, the peripheral sites were often invisible while the juxtanuclear sites appeared as a diffuse blob. If the cells were fixed with organic solvents rather than formaldehyde [4], the results were better but still not satisfactory. Our troubleshooting suggested that cellular architecture was being disrupted by liquid flow

“Corresponding author: Tel. 773-288-5001, Fax 773-702-3172, bsglick@uchicago.edu . 
during the wash steps. We therefore developed an improved immunofluorescence method that incorporates a chemical crosslinking reaction. This method reliably preserves tER sites as well as other cellular structures, including Golgi compartments and microtubules [3].

Our approach is to dehydrate cultured cells and then rehydrate them in a fixative solution. Cells grown on a coverslip are transferred to acetone or methanol at $-20^{\circ} \mathrm{C}$. This treatment appears to extract the lipids almost instantaneously while precipitating the proteins in place [4]. The dehydrated cells are then rehydrated in the presence of a homobifunctional amine-reactive crosslinker. At this point, the sample can be processed using standard procedures for antibody labeling and DNA staining. The crosslinking reaction strongly stabilizes cellular architecture, typically without compromising antibody binding [2,3]. A representative image is shown in Fig. 1, which displays tER sites, microtubules, and nuclear DNA in a dividing cell.

\section{Materials}

\subsection{Coverslips and Wells}

1. No. 1.5 thickness glass coverslips.

2. Material for creating wells on a coverslip. One option is to punch holes in a laminating film sheet such as Cleer Aheer ${ }^{\circledR}$ from C-Line Products (Mt. Prospect, IL), and to attach a piece of the perforated sheet to a standard coverslip. A second option is to use coverslips with wells that are created by a silicone backing, such as SecureSlip ${ }^{\mathrm{TM}}$ coverslips from Grace Bio-Labs (Bend, OR) (see Note 1).

\subsection{Instruments for Processing Coverslips}

1. Fine-point forceps, such as jeweler's microforceps.

2. Rubber hose attached to a vacuum trap.

\subsection{Solutions Made in Advance}

1. Phosphate-buffered saline (10X PBS): dissolve $80 \mathrm{~g} \mathrm{NaCl}, 2.0 \mathrm{~g} \mathrm{KCl}, 14.4 \mathrm{~g}$ $\mathrm{Na}_{2} \mathrm{PO}_{4}$, and $2.4 \mathrm{~g} \mathrm{KH}_{2} \mathrm{PO}_{4}$ in deionized water at a final volume of $1 \mathrm{~L}$. Sterilize by autoclaving, and store at room temperature. Upon 10 -fold dilution, the $\mathrm{pH}$ should be approximately 7.4.

2. $\mathrm{BS}^{3}$ solution (100X): dissolve bis(sulfosuccinimidyl)suberate (Pierce, Rockford, IL) to $10 \mathrm{mM}$ in deionized water. Store at $-80^{\circ} \mathrm{C}$ in $10-\mu \mathrm{L}$ aliquots.

3. Ethylenediamine is prepared as a $100 \mathrm{mM}$ solution. Add $669 \mu \mathrm{L}$ pure ethylenediamine to $90 \mathrm{~mL}$ deionized water. Add $2 \mathrm{~mL} 6 \mathrm{M} \mathrm{HCl}$ to bring the $\mathrm{pH}$ to approximately 8 . Then adjust the $\mathrm{pH}$ to 7.5 with additional $6 \mathrm{M} \mathrm{HCl}$. Adjust to $100 \mathrm{~mL}$ with deionized water. Filter sterilize, and store at $4^{\circ} \mathrm{C}$ protected from light.

4. Mounting solution can be purchased commercially or prepared in the laboratory (see Note 2.) We use the following custom mixture. To $90 \mathrm{~mL}$ glycerol, add $10 \mathrm{~mL}$ of 10X PBS that had been adjusted to $\mathrm{pH} 9$ with $0.5 \mathrm{M} \mathrm{Na}_{2} \mathrm{CO}_{3}$. Dissolve n-propyl gallate in this solution to $5 \%(\mathrm{w} / \mathrm{v})$ using bath sonication. Store at $-80^{\circ} \mathrm{C}$ in $200-\mu \mathrm{L}$ aliquots.

\footnotetext{
$1_{\text {Most of our experiments have employed single-well SecureSlip }}{ }^{\mathrm{TM}}$ coverslips (cat. no. MSR12-0.5), which can be placed in individual wells of a 6-well culture dish. The multi-well versions (such as cat. no. MSR-12) are suitable for placing in a Petri dish.

${ }^{2}$ We have used traditional glycerol-based mounting media, but newer formulations that eliminate refractive index mismatch may significantly improve image quality [5].
} 
5. VALAP: combine an equal weight of paraffin (m.p. $51-53^{\circ} \mathrm{C}$ ), lanolin, and vaseline. Heat and mix until homogeneous. Store in a beaker at room temperature.

6. Normal horse serum (Vector Laboratories, Burlingame, CA) is stored at $4^{\circ} \mathrm{C}$.

7. Gelatin solution: dissolve cell culture grade porcine skin gelatin (Sigma-Aldrich, St. Louis, MO) to $0.1 \%(w / v)$ in deionized water. Filter sterilize and store at room temperature.

8. Hoechst solution (1\%): dissolve Hoechst 33258 (Molecular Probes, Eugene, OR) to $1 \%(\mathrm{w} / \mathrm{v})$ in deionized water. Store at $4^{\circ} \mathrm{C}$ protected from light.

\subsection{Solutions Made Fresh}

1. PBS+: dilute $10 X$ PBS 10 -fold in deionized water, and add $0.1 \%$ n-octyl- $\beta$-Dglucopyranoside (Sigma-Aldrich, St. Louis, MO) (also known as octyl glucosidesee Note 3).

2. Blocking buffer: to $10 \mathrm{~mL}$ PBS+ add $0.1 \mathrm{~g}$ non-fat powdered milk, $222 \mu \mathrm{L} 45 \%$ cold water fish skin gelatin (Sigma-Aldrich, St. Louis, MO), and $0.1 \mathrm{~mL}$ normal horse serum (see Note 4).

3. Antibody solutions: dilute the desired primary and secondary antibodies in blocking buffer. Dilutions typically range from 1:50 to 1:5000 and must be determined empirically for each antibody. Spin $5 \mathrm{~min}$ at maximum speed in a microcentrifuge, and retain the supernatant. If DNA staining is desired, supplement the secondary antibody mixture with a 1:5000 dilution of $1 \%$ Hoechst solution.

\section{Methods}

\subsection{Growth of Cells on Coverslips}

1. If standard glass coverslips are being used, place the sterile coverslips in suitable culture dishes. It may be helpful to etch an asymmetric mark into the top of each coverslip. If SecureSlip ${ }^{\mathrm{TM}}$ coverslips are being used, the coverslips should be precoated with gelatin as follows. Aseptically remove SecureSlip ${ }^{\mathrm{TM}}$ coverslips from the package and place them in a culture dish with the wells facing up. Add $20 \mu \mathrm{L} 0.1 \%$ gelatin to each well. Cover the culture dish and let it sit for $10 \mathrm{~min}$ in the hood. Then aspirate the excess gelatin completely using a Pasteur pipet attached to a vacuum trap. Fill the culture dish with culture medium. Make sure that the SecureSlip ${ }^{\mathrm{TM}}$ coverslips are completely submerged and not floating. If a SecureSlip ${ }^{\mathrm{TM}}$ coverslip does float, push it down to the bottom of the culture dish using a sterile pipette tip or a sterile forceps.

2. Plate the cells at a density that will yield about $60 \%$ confluency on the day of the experiment. Grow the cells in normal culture medium under standard conditions (see Note 5).

\footnotetext{
${ }^{3}$ Octyl glucoside is optional but reduces background labeling with some antibodies. This detergent will not solubilize transmembrane proteins at the concentration used. Octyl glucoside readily absorbs water from the air and should be stored with desiccation. If the octyl glucoside powder was refrigerated, the bottle should be warmed completely to room temperature before opening.

${ }_{5}^{4}$ Other additives can be also used to make a blocking buffer. The formulation given here has worked well for us with a variety of antibodies.

5 The confluency and culture medium can be varied as needed for the purposes of the experiment.
} 


\subsection{Preparations for Immunofluorescence Processing}

1. On the day of the experiment, for each coverslip, fill a 50-ml conical plastic tube with $25 \mathrm{~mL}$ of either acetone or methanol (depending on the antigen-see Note 6). Cool these tubes of organic solvent to $-20^{\circ} \mathrm{C}$.

2. Prepare fresh PBS+ and blocking buffer (see Subheading 2.4).

3. Perform any desired experimental manipulations of the cultured cells.

4. Prepare a humidified chamber for the incubations. A suitable chamber can be created by placing a moist paper towel in a standard Petri dish.

\subsection{Organic Solvent Treatment and Crosslinking}

1. Remove a culture dish from the incubator. Working quickly, lift a coverslip out of the culture dish using forceps (see Note 7).

2. Remove as much culture medium as possible (this point is especially importantsee Note 8). If a standard coverslip is being used, wick away the liquid by touching an edge of the coverslip to a paper towel. If a SecureSlip ${ }^{\mathrm{TM}}$ coverslip is being used, the sides of the well protect the cells, so the culture medium should be removed by inverting the coverlip on a paper towel and pressing gently on the bottom of the coverslip with forceps.

3. Immediately drop the coverslip into a tube of cold acetone or methanol. The goal during these manipulations is to transfer the coverlip from $37^{\circ} \mathrm{C}$ to $-20^{\circ} \mathrm{C}$ as quickly as possible, ideally in less than $5 \mathrm{sec}$.

4. Leave the tube at $-20^{\circ} \mathrm{C}$ for $5 \mathrm{~min}$ (or longer-see Note 9).

5. Remove the coverslip from the organic solvent using forceps. Hold the coverslip vertically in a tissue culture hood until the solvent has completely evaporated. The best results are obtained if the solvent is dried rapidly by touching a corner of the coverslip to the air flow grating.

6. Set the coverslip down on a clean surface with the cells facing up. If desired, the dehydrated cells can be left at room temperature for up to several hours.

7. If a standard coverslip is being used, create wells as follows. Cut a piece of laminating film to about the size of the coverslip. Use a leather punch to make 5-mm holes in the laminating film. Then carefully seal the laminating film to the coverslip by rubbing with a pipette tip. Up to four wells fit readily on a $22 \times 22 \mathrm{~mm}$ square coverslip. If a SecureSlip ${ }^{\mathrm{TM}}$ coverslip is being used, wells are already present on the coverslip (see Note 10).

8. Prepare diluted $\mathrm{BS}^{3}$ crosslinker by adding a $10-\mu \mathrm{L}$ aliquot of $10 \mathrm{mM} \mathrm{BS}^{3}$ to $990 \mu \mathrm{L}$ PBS+. Pipette $10 \mu \mathrm{L}$ of diluted BS ${ }^{3}$ into each well (unless the epitope is sensitive to $\mathrm{BS}^{3}$ treatment-see Note 11). Incubate $30 \mathrm{~min}$ at room temperature in a humidified chamber.

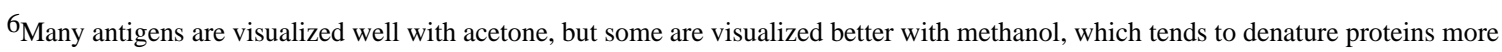
extensively. The choice of organic solvent should be made empirically.

${ }^{7}$ Removing the coverslip from the culture dish can be tricky. The easiest method is to lift one edge of the coverslip with a syringe needle before grabbing the coverslip with forceps.

${ }^{8}$ If a significant amount of culture medium is transferred with the coverslip, the dehydration will initially be incomplete, and the mixture of water and organic solvent will disrupt cellular architecture. It is therefore essential to remove as much as possible of the culture medium before dropping the coverslip into the organic solvent. However, the cells cannot be allowed to dry completely.

9 If desired, coverslips can be left for days or even weeks in organic solvent at $-20^{\circ} \mathrm{C}$.

${ }^{10}$ After prolonged storage of a SecureSlip ${ }^{\mathrm{TM}}$ coverslip in organic solvent, the silicone backing may detach. In this case, dry the coverslip and silicone backing separately, and then reattach the silicone backing, taking care not to perturb the cells.
} 
9. During this incubation, prepare the diluted and centrifuged primary and secondary antibodies (see Subheading 2.4). Prepare enough of each antibody solution to add 10 $\mu \mathrm{L}$ per well.

10. If a standard coverslip is being used, remove the $\mathrm{BS}^{3}$ by aspiration using a Pasteur pipet attached to a vacuum trap. If a SecureSlip ${ }^{\mathrm{TM}}$ coverlip is being used, the preferred method is to blot away the liquid by inverting the coverslip onto a paper towel. In either case, it is important to remove the liquid gently during this 5nd subsequent steps.

11. Wash each well three times with a drop of PBS+. For each wash, add a drop of PBS + to the well, then gently aspirate or blot away the liquid.

12. To quench any unreacted $\mathrm{BS}^{3}$, pipette $10 \mu \mathrm{L}$ of ethylenediamine solution into each well and incubate $15 \mathrm{~min}$ at room temperature (see Note 12).

13. During this incubation, remove particulate matter from the blocking buffer by spinning in a tabletop centrifuge for $15 \mathrm{~min}$ at $2000 \mathrm{xg}$ (typically about $3000 \mathrm{rpm}$ ). Transfer the supernatant to a fresh tube, and use this centrifuged blocking buffer from now on.

\subsection{Antibody Incubations}

1. To block nonspecific binding sites, add a drop of centrifuged blocking buffer to each well. Incubate $1 \mathrm{~h}$ at room temperature.

2. Gently aspirate or blot away the blocking buffer. Add $10 \mu \mathrm{L}$ of primary antibody solution to each well. Incubate 30-60 min at room temperature.

3. Gently wash each well 8 times with a drop of blocking buffer.

4. Add $10 \mu \mathrm{L}$ of secondary antibody solution to each well. Incubate $30 \mathrm{~min}$ at room temperature.

5. Gently wash each well 10 times with a drop of blocking buffer.

\subsection{Mounting the Samples}

1. Completely aspirate the final drop of blocking buffer from each well. It is essential that no liquid remains (see Note 13).

2. Add $10 \mu \mathrm{L}$ of mounting solution to each well (see Note 2).

3. Invert the coverslip onto a glass slide. If a standard coverslip is being used, seal the edges with VALAP. If a SecureSlip ${ }^{\mathrm{TM}}$ coverslip is being used, seal the edges with clear nail polish.

4. If possible, the samples should be viewed immediately, but good results can be obtained for several weeks if the slides are stored at room temperature in the dark.

\footnotetext{
${ }^{11}$ Some epitopes might be blocked by treatment with $\mathrm{BS}^{3}$, which reacts mainly with lysine side chains. If the staining is weak after $\mathrm{BS}^{3}$ treatment, rehydration can be done with PBS+ lacking $\mathrm{BS}^{3}$. In this case, the washes should be exceptionally gentle to avoid disrupting cellular architecture.

12 Ethylenediamine is a very potent quencher of amine-reactive crosslinkers. More common quenchers such as glycine would presumably also be effective, but the incubation period might need to be prolonged.

${ }^{13}$ If any droplets of liquid remain, they may not mix completely with the mounting solution, and the resulting refractive index mismatch will distort the images.
} 


\section{Acknowledgments}

This work was supported by NIH grant GM-61156. The anti-Sec13 antibody was a kind gift of Bor Luen Tang and Wanjin Hong (National University of Singapore).

\section{References}

1. Donaldson, JG. Current Protocols in Cell Biology. John Wiley \& Sons; 1998. Immunofluorescence Staining; p. 4.3.1-4.3.6.

2. Bhattacharyya D, Glick BS. Two mammalian Sec16 homologs have nonredundant functions in ER export and transitional ER organization. Mol. Biol. Cell 2007 18;:839-849. [PubMed: 17192411]

3. Hammond AT, Glick BS. Dynamics of transitional endoplasmic reticulum sites in vertebrate cells. Mol. Biol. Cell 2000;11:3013-3030. [PubMed: 10982397]

4. Melan MA, Sluder G. Redistribution and differential extraction of soluble proteins in permeabilized cultured cells. Implications for immunofluorescence microscopy. J. Cell Sci 1992;101:731-743. [PubMed: 1527176]

5. Staudt T, Lang MC, Medda R, Engelhardt J, Hell SW. 2,2'-Thiodiethanol: a new water soluble mounting medium for high resolution optical microscopy. Microsc. Res. Tech 2007;70:1-9. [PubMed: 17131355]

6. Tang BL, et al. The mammalian homolog of yeast Sec13p is enriched in the intermediate compartment and is essential for protein transport from the endoplasmic reticulum to the Golgi apparatus. Mol. Cell. Biol 1997;17:256-266. [PubMed: 8972206] 


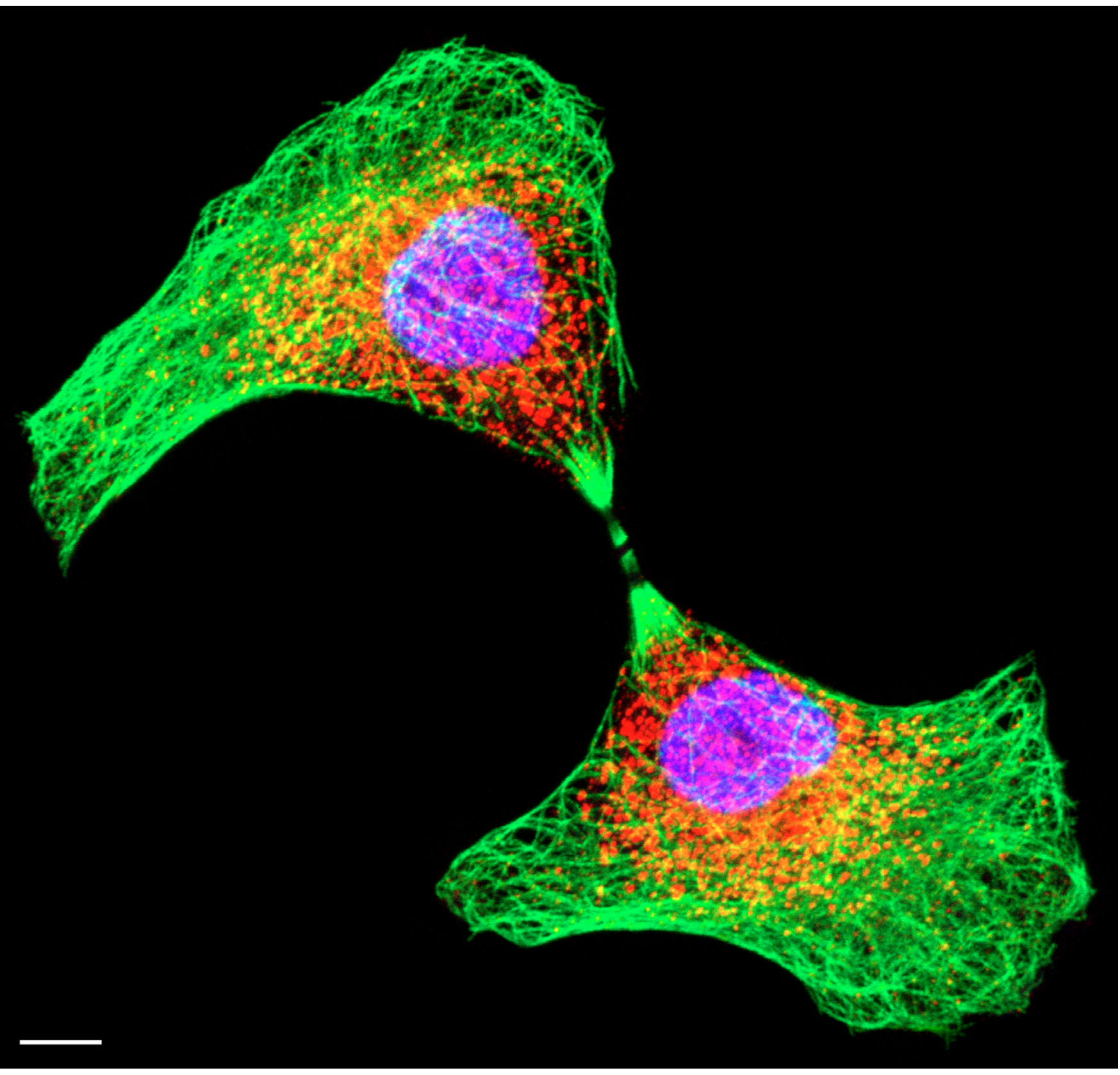

Figure 1.

Immunofluorescence image of a dividing normal rat kidney cell. Microtubules (green) were stained with a monoclonal anti- $\beta$-tubulin antibody (clone KMX-1, Roche, Indianapolis, IN) followed by Cy2-conjugated donkey antimouse antibody. tER sites (red) were stained with an affinity-purified polyclonal anti-Sec13 antibody [6] followed by Rhodamine Red-Xconjugated donkey anti-rabbit antibody. Both primary antibodies were diluted 1:100, and both secondary antibodies (from Jackson Immunoresearch, West Grove, PA) were diluted 1:200. DNA (magenta) was stained by supplementing the mounting medium with $4 \mathrm{mM}$ TOTO-3 (Molecular Probes, Eugene, OR). Separate Z-stacks in three fluorescence channels were collected with a Zeiss (Thornwood, NY) LSM 510 confocal microscope equipped with a 100X 1.4-NA Plan-Apo objective and with standard filters for visualizing FITC/Cy2, Rhodamine Red-X, and Cy5/TOTO-3. These images were then projected and combined using the Zeiss 
software. The background staining outside of the cells was removed using Adobe Photoshop. Scale bar, $10 \mu \mathrm{m}$. 